\title{
Glauber gluons in annihilation amplitudes for heavy meson decays
}

\author{
Hsiang-nan Li谓 \\ ${ }^{1}$ Institute of Physics, Academia Sinica, Taipei, Taiwan 115, Republic of China,
}

(Dated: June 9, 2021)

\begin{abstract}
We investigate the Glauber divergences in nonfactorizable annihilation amplitudes for two-body hadronic heavy meson decays in the $k_{T}$ factorization theorem at one-loop level. These divergences can be absorbed into the Glauber factors in the dominant kinematic regions of small parton momenta, which modify the interference between a nonfactorizable annihilation amplitude and other amplitudes by rotating it with a phase. We postulate that only the Glauber effect associated with a pion is significant, due to its special role as a $q \bar{q}$ bound state and as a pseudo Nambu-Goldstone boson simultaneously. It is elaborated that the data of the $D \rightarrow \pi \pi$ and $\pi K$ branching ratios have revealed prominent Glauber effects. This work provides a solid theoretical ground for the factorization-assisted topological-amplitude parametrization of two-body hadronic $D$ meson decays.
\end{abstract}

PACS numbers:

\section{INTRODUCTION}

The existence, factorization, and impact of a special type of infrared divergences, called Glauber gluons [1 $[\underline{6}]$, on studies of two-body hadronic heavy meson decays have been explored thoroughly [7]. It was pointed out that the puzzles from the $B \rightarrow \pi \pi$ and $\pi \rho$ branching ratios, and from the $B \rightarrow \pi K$ direct $\mathrm{CP}$ asymmetries are attributed to the color-suppressed tree amplitudes [8-10]. We then analyzed radiative corrections to the spectator diagrams for these amplitudes involved in two-body hadronic $B$ meson decays $B \rightarrow M_{1} M_{2}$ [7], where $M_{2}$ denotes the meson emitted at the weak vertex. It was found that the Glauber divergences are produced in the $k_{T}$ factorization theorem [11, 12], and can be absorbed into additional nonperturbative factors in the dominant kinematic regions with small parton momenta [13]. The all-order organization of the Glauber divergences follows the standard factorization procedure [14], which relies on the eikonal approximation for soft gluons. The resultant Glauber phase factor $\exp \left(-i S_{e 1}\right)$ associated with the $M_{1}$ meson is the same for the two leading-order (LO) spectator diagrams [15]. The Glauber factors from $M_{2}$ carry opposite phases, namely, $\exp \left(i S_{e 2}\right)$ for one diagram, and $\exp \left(-i S_{e 2}\right)$ for another [7]. Therefore, they have different impacts on a spectator amplitude: the latter enhances the spectator contribution by modifying the interference pattern between the two LO diagrams. The former rotates the enhanced spectator contribution by a phase, and changes its interference with other amplitudes in two-body hadronic $B$ meson decays.

We postulated that only the Glauber factors associated with a pion give significant effects, due to its special role as a $q \bar{q}$ bound state and as a pseudo Nambu-Goldstone (NG) boson simultaneously [16]. The Glauber factors $\exp \left(-i S_{e 1}\right)$ for $M_{1}$ and $\exp \left( \pm i S_{e 2}\right)$ for $M_{2}$ were introduced into the spectator amplitudes and treated as additional inputs [17, 18] in the PQCD approach based on the $k_{T}$ factorization theorem. Though a Glauber factor is universal, it causes different effects through its convolution with various transverse-momentum-dependent (TMD) meson wave functions. It turned out that the Glauber effects from a pion are indeed stronger, and improve the consistency between PQCD predictions and experimental data for all the $B \rightarrow \pi M$ decays with $M=\pi, \rho$ and $K[17,18]$. In particular, the rotation of the spectator amplitude by $\exp \left(-i S_{e 1}\right)$ is crucial for enhancing the ratio of the $B^{+} \rightarrow \pi^{+} \pi^{0}$ branching fraction over the $B^{0} \rightarrow \pi^{+} \pi^{-}$one: this ratio depends on both the color-allowed tree amplitude and the color-suppressed tree amplitude, so the relative phase between them matters. It is a nontrivial success that all the puzzles in the $B$ meson decays mentioned before were resolved at the same time by introducing two Glauber phases.

The dramatic distinction between the measured $D^{0} \rightarrow \pi^{+} \pi^{-}$and $D^{0} \rightarrow K^{+} K^{-}$branching ratios represented another salient puzzle from $D$ meson decays: the former (latter) is lower (higher) than theoretical predictions from analyses based on the topological-amplitude parametrization [19, 20]. The deviation in

*Electronic address: hnli@phys.sinica.edu.tw 


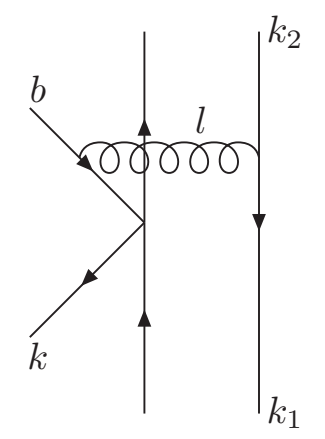

(a)

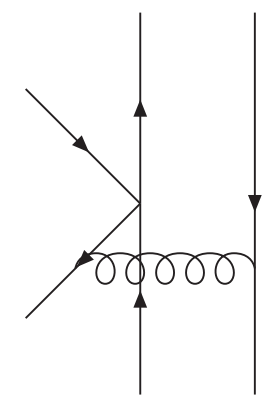

(b)

FIG. 1: LO diagrams for a nonfactorizable annihilation amplitude.

these two modes stands even after flavor SU(3) symmetry breaking effects in emission amplitudes were taken into account [20]. This subject was investigated in the factorization-assisted topological-amplitude (FAT) approach [21], where the Glauber phase was introduced into the nonfactorizable annihilation amplitudes for two-body hadronic $D$ meson decays. This additional phase modifies the interference between the annihilation and emission amplitudes involving pions, and improves the overall agreement with data by decreasing the predicted $D^{0} \rightarrow \pi^{+} \pi^{-}$branching ratio. Once the Galuber phase was fixed in a global fit to measured branching ratios in the FAT framework, the penguin amplitudes, expressed as the combination of the determined hadronic parameters and the corresponding Wilson coefficients, were also obtained accordingly. We then predicted direct $\mathrm{CP}$ asymmetries in $D \rightarrow P P$ decays, with $P$ denoting a pseudoscalar meson, in the Standard Model without ambiguity. Especially, we predicted the difference between the two direct $\mathrm{CP}$ asymmetries, $\Delta A_{\mathrm{CP}} \equiv A_{\mathrm{CP}}\left(D^{0} \rightarrow K^{+} K^{-}\right)-A_{\mathrm{CP}}\left(D^{0} \rightarrow \pi^{+} \pi^{-}\right) \approx-1.0 \times 10^{-3}$ [21], which was verified by the more precise $\mathrm{LHCb}$ data [22, 23] announced later. We mention that a prediction for $\Delta A_{\mathrm{CP}}$ similar to ours was made in the topological-amplitude approach combined with final-state rescattering [24].

However, the existence and factorization of the Glauber divergences in the nonfactorizable annihilation diagrams were not verified rigorously in Ref. [21]. Here we will explore the Glauber gluons in these diagrams in the $k_{T}$ factorization theorem, and show that they can be factorized into the nonperturbative phase factors associated with final-state mesons in the kinematic regions with small parton momenta. It is found that the Glauber phases are the same for the two LO nonfactorizable annihilation diagrams, so the Glauber effect rotates a nonfactorizable annihilation amplitude, and modifies its interference with other amplitudes. To reveal the Glauber effects in two-body hadronic $D$ meson decays, we highlight several $D \rightarrow \pi \pi$ and $\pi K$ modes, whose measured branching ratios demand the inclusion of the Glauber phases in the FAT approach. This work provides a solid theoretical ground for the FAT parametrization, and confirms the speculation that the Glauber effects associated with a pion are crucial for resolving the puzzles in heavy meson decays [25].

In Sec. II we analyze the collinear divergences in radiative corrections to the nonfactorizable annihilation amplitudes in the $k_{T}$ factorization theorem at one-loop level, and identify the residual infrared divergences caused by the Glauber gluons that cannot be absorbed into TMD meson wave functions. The Glauber divergences are factorized out of the two LO nonfactorizable annihilation diagrams and grouped into the nonperturbative phase factors. In Sec. III we discuss the data for the $D \rightarrow \pi \pi$ and $D \rightarrow \pi K$ branching ratios, which exhibit the apparent Glauger effects. Section IV contains the conclusion.

\section{FACTORIZATION OF GLAUBER GLUONS}

We examine the infrared divergences in the next-to-leading-order (NLO) nonfactorizable annihilation diagrams, following the reasoning in Ref. [7]. Consider the $B\left(P_{B}\right) \rightarrow M_{1}\left(P_{1}\right) M_{2}\left(P_{2}\right)$ decay, where $P_{B}, P_{1}$, and $P_{2}$ are the momenta of the $B, M_{1}$, and $M_{2}$ mesons, respectively. For convenience, we choose $P_{B}=\left(P_{B}^{+}, P_{B}^{-}, \mathbf{0}_{T}\right)$ with $P_{B}^{+}=P_{B}^{-}=m_{B} / \sqrt{2}, m_{B}$ being the $B$ meson mass, and $P_{1}\left(P_{2}\right)$ in the plus (minus) direction. The parton momenta $k, k_{1}$ and $k_{2}$ for the $B, M_{1}$ and $M_{2}$ mesons, respectively, are labelled in Fig. 1(a), which are assumed 


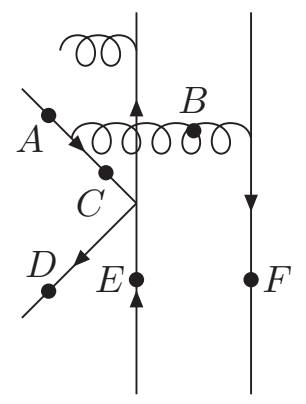

$(a)$

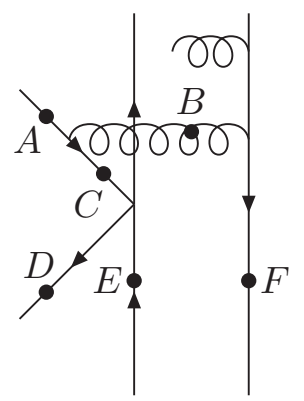

(b)

FIG. 2: NLO diagrams for Fig. 1 (a), where the radiative gluon is emitted by (a) the valence quark and (b) the valence anti-quark of the $M_{2}$ meson, and attaches to the vertices labelled by $A, B, \ldots$, or $F$.

to obey the hierarchy in the regions of small parton momenta [26],

$$
m_{B} \gg k_{1}^{+}, k_{2}^{-} \gg k^{\mu}, k_{i T} \sim O(\Lambda),
$$

with $i=1,2$ and $\Lambda$ representing a small scale. The even smaller components $k_{1}^{-}$and $k_{2}^{+}$under the above hierarchy have been neglected.

\section{A. NLO Corrections to Fig. 1)(a)}

We first search for the Glauber gluons associated with the LO nonfactorizable annihilation diagram in Fig. 1(a), starting with the set of NLO diagrams in Fig. 2. Due to the soft cancellation between the gluons radiated by the valence quark and by the valence anti-quark of the $M_{2}$ meson 27], only the collinear region with the loop momentum $l$ being collimated to $P_{2}$ is relevant, and the particles in the $B$ and $M_{1}$ mesons, to which the collinear gluons attach, are off-shell. The transverse-momentum dependence of these propagators is thus negligible, which can then be approximated by the eikonal propagators $1 /\left(l^{-} \pm i \epsilon\right)$. For a loop diagram to generate an imaginary Glauber logarithm, a necessary (but not sufficient) condition is that the interval of $l^{-}$ covers the origin $l^{-}=0$. The corresponding integrals then contain imaginary pieces,

$$
\operatorname{Im} \int \frac{d l^{-}}{l^{-} \pm i \epsilon}=\mp \pi \int d l^{-} \delta\left(l^{-}\right)=\mp \pi
$$

under the principal-value prescription. As the radiative gluon goes on shell, an imaginary logarithm appears. It is known that a TMD meson wave function does not involve imaginary infrared logarithms. Hence, the Glauber logarithm is residual, and cannot be absorbed into the $M_{2}$ meson wave function in view of its universality.

Start with Fig. 2(a), where the radiative gluon is emitted by the valence quark of the $M_{2}$ meson and attaches to the other lines. The reducible diagrams, such as the self-energy correction to the valence quark of $M_{2}$, are not displayed. For the attachments $A, C, D$ and $E$, the pole structures of the loop integrands on the $l^{+}$plane imply that only the $l^{-}<0$ range contributes to the contour integrals over $l^{+}$. Taking the attachment $C$ as an example, we have the integrand proportional to

$$
\frac{1}{2\left(l^{-}+\bar{k}_{2}^{-}\right) l^{+}-\left|\mathbf{k}_{2 T}-\mathbf{l}_{T}\right|^{2}+i \epsilon} \frac{1}{2 l^{-} l^{+}-l_{T}^{2}+i \epsilon} \frac{1}{2\left(l^{-}+\bar{k}_{2}^{-}\right)\left(l^{+}+\bar{k}_{1}^{+}\right)-m_{b}^{2}+i \epsilon},
$$

where $\bar{k}_{1} \equiv P_{1}-k_{1}, \bar{k}_{2}=P_{2}-k_{2}$, and the $k^{ \pm}$and TMD terms in the hard $b$ quark propagator have been dropped based on Eq. (1) for simplicity. When poles are located in the different half planes of $l^{+}$, the loop integral does not vanish, no matter whether the contour of $l^{+}$is closed from the upper or lower half plane. It is the case for Eq. (3) only when $l^{-}<0$ obviously. Then the attachment $C$ does not contribute a Glauber 
divergence according to Eq. (2). A general observation is that a simple diagram like a vertex correction, ie., the attachment $C, D$ or $E$, does not develop a Glauber divergence.

The attachment $B$, with the radiative gluon attaching to a hard gluon, does not produce a Glauber divergence either. The corresponding integrand proportional to

$$
\begin{aligned}
& \frac{1}{2\left(l^{-}+\bar{k}_{2}^{-}\right) l^{+}-\left|\mathbf{k}_{2 T}-\mathbf{l}_{T}\right|^{2}+i \epsilon} \frac{1}{2 l^{-} l^{+}-l_{T}^{2}+i \epsilon} \frac{1}{2\left(l^{-}-k_{2}^{-}\right)\left(l^{+}-k_{1}^{+}\right)-\left|\mathbf{k}_{1 T}+\mathbf{k}_{2 T}-\mathbf{l}_{T}\right|^{2}+i \epsilon} \\
& \times \frac{1}{2\left(l^{-}+\bar{k}_{2}^{-}\right)\left(l^{+}+\bar{k}_{1}^{+}\right)-m_{b}^{2}+i \epsilon},
\end{aligned}
$$

indicates that the loop integral does not vanish as $-\bar{k}_{2}^{-}<l^{-}<k_{2}^{-}$. We focus on the poles in the lower half plane of $l^{+}$. The pole $l^{+}=l_{T}^{2} /\left(2 l^{-}\right)$of the second, ie., radiative gluon propagator moves from the lower half plane to the upper one, when $l^{-}$varies from $l^{-}>0$ to $l^{-}<0$. That is, its residue does not exist in both the positive and negative regions of $l^{-}$required by Eq. (2). The pole $l^{+}=m_{b}^{2} /\left[2\left(l^{-}+\bar{k}_{2}^{-}\right)\right]-\bar{k}_{1}^{+}$of the last, ie., $b$ quark propagator yields an off-shell quark in the $M_{2}$ meson denoted by the first propagator. Namely, it does not contribute a collinear configuration associated with $M_{2}$. We thus pick up the pole $l^{+}=\left|\mathbf{k}_{2 T}-\mathbf{l}_{T}\right|^{2} /\left[2\left(l^{-}+\bar{k}_{2}^{-}\right)\right] \sim O\left(\Lambda^{2} / m_{B}\right)$ from the first propagator, which is much smaller than $m_{B}$ and makes a collinear configuration. However, the denominators for both the hard gluon corresponding to the third propagator and the hard $b$ quark do not flip sign in the range $-\bar{k}_{2}^{-}<l^{-}<k_{2}^{-}$, and thus no imaginary pieces are generated.

We conclude that the attachments $A-E$ in Fig. 2(a) contribute only to the $M_{2}$ meson wave function, whose collinear divergences can be collected by the Wilson links resulting from the eikonal approximation. The detailed procedure for factorizing these NLO diagrams is referred to [14].

Possible Glauber divergences may appear in the attachment $F$, whose integrand contains the denominator

$$
\left[\left(\bar{k}_{2}+l\right)^{2}+i \epsilon\right]\left[\left(\bar{k}_{1}+\bar{k}_{2}-k+l\right)^{2}-m_{b}^{2}+i \epsilon\right]\left(l^{2}+i \epsilon\right)\left[\left(k_{1}-l\right)^{2}+i \epsilon\right]\left[\left(k_{1}+k_{2}-l\right)^{2}+i \epsilon\right] .
$$

Nonvanishing contributions come from the range $0<l^{-}<k_{2}^{-}\left(-\bar{k}_{2}^{-}+k^{-}<l^{-}<0,-\bar{k}_{2}^{-}<l^{-}<-\bar{k}_{2}^{-}+k^{-}\right)$, where the poles of $l^{+}$are given by

$$
\begin{aligned}
& l^{+}=\frac{\left|\mathbf{l}_{T}-\mathbf{k}_{2 T}\right|^{2}}{2\left(l^{-}+\bar{k}_{2}^{-}\right)}-i \epsilon(-i \epsilon,-i \epsilon), \\
& l^{+}=-\bar{k}_{1}^{+}+k^{+}+\frac{m_{b}^{2}}{2\left(l^{-}+\bar{k}_{2}^{-}-k^{-}\right)}-i \epsilon(-i \epsilon,+i \epsilon), \\
& l^{+}=\frac{l_{T}^{2}}{2 l^{-}}-i \epsilon(+i \epsilon,+i \epsilon), \\
& l^{+}=k_{1}^{+}+\frac{\left|\mathbf{l}_{T}-\mathbf{k}_{1 T}\right|^{2}}{2 l^{-}}-i \epsilon(+i \epsilon,+i \epsilon), \\
& l^{+}=k_{1}^{+}+\frac{\left|\mathbf{l}_{T}-\mathbf{k}_{1 T}-\mathbf{k}_{2 T}\right|^{2}}{2\left(l^{-}-k_{2}^{-}\right)}+i \epsilon(+i \epsilon,+i \epsilon) .
\end{aligned}
$$

We pick up the poles in the lower half plane of $l^{+}$. The first pole $l^{+} \sim O\left(\Lambda^{2} / m_{B}\right)$ contributes to the loop integral in the range $-\bar{k}_{2}^{-}<l^{-}<k_{2}^{-}$, which covers the origin $l^{-}=0$, and also makes a collinear configuration associated with the $M_{2}$ meson. It is then seen that the loop integral develops a Glauber divergence from the eikonalized spectator propagator $1 /\left(k_{1}-l\right)^{2}$ and the on-shell radiative gluon. The second pole $l^{+} \sim O\left(k_{1}^{+}\right)$ in Eq. (7), contributing to the loop integral in the range $-\bar{k}_{2}^{-}+k^{-}<l^{-}<k_{2}^{-}$, does not correspond to the considered collinear configuration.

An alternative way to verify the existence of the Glauber divergence in the attachment $F$ follows the derivation in Ref. [1]: we eikonalize the quark line with the momentum $k_{1}-l$ first, and focus on the imaginary piece proportional to $\delta\left(l^{-}\right)$. The integration over $l^{-}$leads the radiative gluon propagator to $1 / l_{T}^{2}$, and the quark propagator on the $M_{2}$ side and the hard gluon propagator to

$$
\frac{1}{2 \bar{k}_{2}^{-} l^{+}-\left|\mathbf{k}_{2 T}-\mathbf{l}_{T}\right|^{2}+i \epsilon} \frac{1}{2 k_{2}^{-}\left(k_{1}^{+}-l^{+}\right)-\left|\mathbf{k}_{1 T}+\mathbf{k}_{2 T}-\mathbf{l}_{T}\right|^{2}+i \epsilon} .
$$

We do not show the hard $b$ quark propagator, since its $l$ dependence does not affect the conclusion. The above expression implies clearly that the contour integral over $l^{+}$does not vanish, because the two poles of $l^{+}$are 
located on different half planes, and that the Glauber divergence from $l_{T} \rightarrow 0$ stands. It is also easy to explain by means of Eq. (11) that a Glauber divergence does not appear in the collinear factorization theorem for twobody hadronic $B$ meson decays [28], which assumes the dominance of the region with large parton momenta $k_{1}^{+} \sim k_{2}^{-} \sim O\left(m_{B}\right)$. The small component $l^{+}$in the collinear configuration is then negligible in the hard gluon and $b$ quark propagators, such that only a single pole of $l^{+}$from the first propagator in Eq. (11) remains, and the contour integral over $l^{+}$vanishes. In other words, a Glauber divergence is power suppressed in the collinear factorization.

We then study the infrared divergences from Fig. 2(b) with the radiative gluon being emitted by the valence anti-quark of the $M_{2}$ meson and attaching to the other lines. Similarly, for the attachments $A, B$ and $F$, the pole structures of the loop integrands on the $l^{+}$plane indicate that only the $l^{-}<0$ range contributes to the contour integrals over $l^{+}$. The radiative gluon attaches to the hard $b$ quark in the attachment $C$. As elaborated before, no Glauber divergence exists in the above cases.

The integrand for the attachment $D$ has five denominators,

$$
\left[\left(k_{1}+k_{2}+l\right)^{2}+i \epsilon\right]\left[\left(k_{2}+l\right)^{2}+i \epsilon\right]\left[(k+l)^{2}+i \epsilon\right]\left(l^{2}+i \epsilon\right)\left[\left(\bar{k}_{1}+\bar{k}_{2}-k-l\right)^{2}-m_{b}^{2}+i \epsilon\right] .
$$

Nonvanishing contributions arise from the range $0<l^{-}<\bar{k}_{2}^{-}-k^{-}\left(-k^{-}<l^{-}<0,-k_{2}^{-}<l^{-}<-k^{-}\right)$, where the poles of $l^{+}$are given by

$$
\begin{aligned}
l^{+} & =-k_{1}^{+}+\frac{\left|\mathbf{l}_{T}+\mathbf{k}_{1 T}+\mathbf{k}_{2 T}\right|^{2}}{2\left(l^{-}+k_{2}^{-}\right)}-i \epsilon(-i \epsilon,-i \epsilon), \\
l^{+} & =\frac{\left|\mathbf{l}_{T}+\mathbf{k}_{2 T}\right|^{2}}{2\left(l^{-}+k_{2}^{-}\right)}-i \epsilon(-i \epsilon,-i \epsilon), \\
l^{+} & =-k^{+}+\frac{\left|\mathbf{l}_{T}+\mathbf{k}_{T}\right|^{2}}{2\left(l^{-}+k^{-}\right)}-i \epsilon(-i \epsilon,+i \epsilon), \\
l^{+} & =\frac{l_{T}^{2}}{2 l^{-}}-i \epsilon(+i \epsilon,+i \epsilon), \\
l^{+} & =\bar{k}_{1}^{+}-k^{+}+\frac{m_{b}^{2}}{2\left(l^{-}-\bar{k}_{2}^{-}+k^{-}\right)}+i \epsilon(+i \epsilon,+i \epsilon) .
\end{aligned}
$$

It is more convenient to pick up the poles in the upper half plane of $l^{+}$, and noticed that only the last pole contributes in the range $-k_{2}^{-}<l^{-}<\bar{k}_{2}^{-}-k^{-}$covering the origin $l^{-}=0$. However, this pole sets a large $l^{+}$ component, with which the other four propagators, being off-shell, do not generate Glauber divergences.

The poles involved in the attachment $E$ are similar to those in the attachment $D$, but with the third one in Eq. (15) being replaced by

$$
l^{+}=\bar{k}_{1}^{+}+\frac{\left|\mathbf{l}_{T}+\mathbf{k}_{1 T}\right|^{2}}{2 l^{-}}-i \epsilon(+i \epsilon,+i \epsilon) .
$$

The same argument applies to this case apparently, and we conclude that the attachment $E$ does not yield a Glauber divergence either. That is, the diagrams in Fig. 2(b) contribute only to the $M_{2}$ meson wave function, whose collinear divergences can be collected by the Wilson links.

The eikonalization of the propagator $1 /\left[\left(k_{1}-l\right)^{2}+i \epsilon\right]$ for the attachment $F$ in Fig. 2 (a) leads to $1 /\left(-l^{-}+i \epsilon\right)$ [7], which contributes an imaginary piece $-\pi i \delta\left(l^{-}\right)$. The principal value, ie., the real piece of this eikonal propagator gives rise to a Wilson link, which goes into the definition of the $M_{2}$ meson wave function [14]. The NLO residual infrared divergence is then written as

$$
\begin{aligned}
& -\frac{g^{2}}{2 N_{c}} \int \frac{d^{4} l}{(2 \pi)^{4}} \operatorname{tr}\left[\ldots \gamma_{5} \not P_{2}\left(-i g \gamma^{-}\right) \frac{i\left(\bar{k}_{2}+\not l\right)}{\left(k_{2}+l\right)^{2}+i \epsilon}\right] \\
& \times \frac{-i}{\left(k_{1}+k_{2}-l\right)^{2}+i \epsilon} \frac{-i}{l^{2}+i \epsilon}(-\pi i) \delta\left(l^{-}\right),
\end{aligned}
$$

where ... denotes the rest of the integrand, and $\gamma_{5} \not P_{2}$ comes from the twist- 2 structure of the $M_{2}$ meson wave function. Note that this Glauber divergence carries the color factor $-1 /\left(2 N_{c}\right)$ [27], $N_{c}$ being the number of 


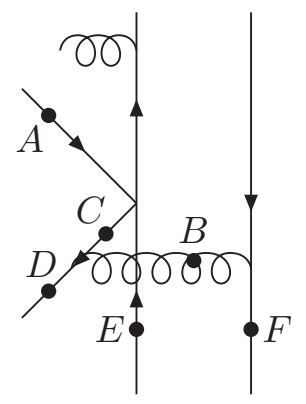

$(a)$

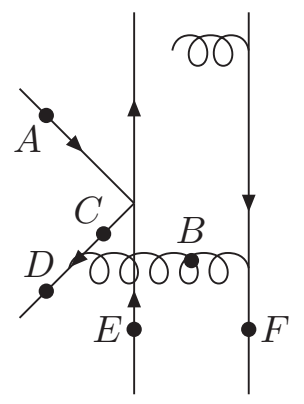

(b)

FIG. 3: NLO diagrams for Fig. 1(b), where the radiative gluon is emitted by (a) the valence quark and (b) the valence anti-quark of the $M_{2}$ meson, and attaches to the vertices labelled by $A, B, \ldots$, or $F$.

colors. Because the pole $l^{+} \sim O\left(k_{1}^{+}\right)$in Eq. (10) in the upper half plane stays far away from the selected pole $l^{+} \sim O\left(\Lambda^{2} / m_{B}\right)$, we can always deform the contour of $l^{+}$, such that $l^{+}$remains at least $O(\Lambda)$, and the hierarchy

$$
\bar{k}_{2}^{-} l^{+} \sim O\left(m_{B} \Lambda\right) \gg\left|\mathbf{l}_{T}-\mathbf{k}_{2 T}\right|^{2} \sim O\left(\Lambda^{2}\right),
$$

holds. The quark with the momentum $\bar{k}_{2}+l$ in Eq. (19) can then be eikonalized into $1 /\left(l^{+}+i \epsilon\right)$. The above argument goes exactly like that applied to the factorization of the Glauber gluons from the low- $p_{T}$ hadron hadroproduction [13].

Equation (19) is thus factorized into

$$
\begin{aligned}
& \frac{g^{2}}{2 N_{c}} \int \frac{d^{4} l}{(2 \pi)^{4}} \operatorname{tr}\left[\ldots \gamma_{5} \not P_{2}\right] \frac{-i}{\left(k_{1}+k_{2}+l\right)^{2}+i \epsilon} \\
& \times \frac{1}{l^{+}+i \epsilon} \frac{-i}{l^{2}+i \epsilon} \pi i \delta\left(l^{-}\right) .
\end{aligned}
$$

We close the contour in the lower half plane of $l^{+}$, pick up the pole $l^{+}=0-i \epsilon$ from the eikonal propagator $1 /\left(l^{+}+i \epsilon\right)$, and derive the convolution in the transverse momentum of the Glauber gluon,

$$
\frac{i}{2 N_{c}} \frac{\alpha_{s}}{2 \pi} \int \frac{d^{2} l_{T}}{l_{T}^{2}} \mathcal{M}_{a}^{(0)}\left(\mathbf{l}_{T}\right)
$$

The imaginary logarithm is explicit in the above expression, and $\mathcal{M}_{a}^{(0)}$ denotes the LO nonfactorizable annihilation amplitude from Fig. 1(a).

\section{B. NLO Corrections to Fig. 1 (b)}

We search for the Glauber divergences in the NLO corrections to Fig. 1(b), which include the attachments of the radiative gluon emitted by the valence quark of the $M_{2}$ meson as depicted in Fig. 3(a). The attachments $A$, $C$ and $E$, being the simple vertex corrections without Glauber divergences, contribute only to the $M_{2}$ meson wave function. The attachment $B$ on the hard gluon does not produce a Glauber divergence. We then discuss the attachment $D$, whose integrand is proportional to

$$
\begin{aligned}
& \frac{1}{2\left(l^{-}+\bar{k}_{2}^{-}\right) l^{+}-\left|\mathbf{k}_{2 T}-\mathbf{l}_{T}\right|^{2}+i \epsilon} \frac{1}{2 l^{-} l^{+}-l_{T}^{2}+i \epsilon} \frac{1}{2\left(l^{-}+k^{-}\right)\left(l^{+}+k^{+}\right)-\left|\mathbf{k}_{T}+\mathbf{l}_{T}\right|^{2}+i \epsilon} \\
& \times \frac{1}{2\left(l^{-}+k^{-}-k_{2}^{-}\right)\left(l^{+}+k^{+}-k_{1}^{+}\right)-\left|\mathbf{k}_{T}-\mathbf{k}_{1 T}-\mathbf{k}_{2 T}+\mathbf{l}_{T}\right|^{2}+i \epsilon} .
\end{aligned}
$$


Similar to the attachment $D$ in Fig. 2(b), it is more convenient to pick up the poles in the upper half plane of $l^{+}$, and noticed that only the last pole contributes in the range $-\bar{k}_{2}^{-}<l^{-}<k_{2}^{-}-k^{-}$covering the origin $l^{-}=0$. However, this pole specifies a large $l^{+} \approx k_{1}^{+}$component, with which the other three propagators stay off-shell, and do not generate Glauber divergences.

The integrand for the attachment $F$ in Fig. 3(a) has the denominator

$$
\left[\left(\bar{k}_{2}+l\right)^{2}+i \epsilon\right]\left(l^{2}+i \epsilon\right)\left[\left(k_{1}-l\right)^{2}+i \epsilon\right]\left[\left(k-k_{1}-k_{2}+l\right)^{2}+i \epsilon\right]\left[\left(k_{1}+k_{2}-l\right)^{2}+i \epsilon\right] .
$$

Nonvanishing contributions come from the range $k_{2}^{-}-k^{-}<l^{-}<k_{2}^{-}\left(0<l^{-}<k_{2}^{-}-k^{-},-\bar{k}_{2}^{-}<l^{-}<0\right)$, where the poles of $l^{+}$are given by

$$
\begin{aligned}
& l^{+}=\frac{\left|\mathbf{l}_{T}-\mathbf{k}_{2 T}\right|^{2}}{2\left(l^{-}+\bar{k}_{2}^{-}\right)}-i \epsilon(-i \epsilon,-i \epsilon), \\
& l^{+}=\frac{l_{T}^{2}}{2 l^{-}}-i \epsilon(-i \epsilon,+i \epsilon), \\
& l^{+}=k_{1}^{+}+\frac{\left|\mathbf{l}_{T}-\mathbf{k}_{1 T}\right|^{2}}{2 l^{-}}-i \epsilon(-i \epsilon,+i \epsilon), \\
& l^{+}=k_{1}^{+}-k^{+}+\frac{\left|\mathbf{l}_{T}-\mathbf{k}_{1 T}-\mathbf{k}_{2 T}+\mathbf{k}_{T}\right|^{2}}{2\left(l^{-}-k_{2}^{-}+k^{-}\right)}-i \epsilon(+i \epsilon,+i \epsilon), \\
& l^{+}=k_{1}^{+}+\frac{\left|\mathbf{l}_{T}-\mathbf{k}_{1 T}-\mathbf{k}_{2 T}\right|^{2}}{2\left(l^{-}-k_{2}^{-}\right)}+i \epsilon(+i \epsilon,+i \epsilon) .
\end{aligned}
$$

We pick up the poles in the lower half plane of $l^{+}$, and find that only the first pole $l^{+} \sim O\left(\Lambda_{\mathrm{QCD}}^{2} / m_{B}\right)$ contributes to the loop integral in the range $-\bar{k}_{2}^{-}<l^{-}<k_{2}^{-}$covering the origin $l^{-}=0$. It is also the pole that corresponds to the considered collinear configuration associated with the $M_{2}$ meson. It is seen that the loop integral develops a Glauber divergence from the eikonalized quark propagator $1 /\left(k_{1}-l\right)^{2}$ and the on-shell radiative gluon, with the color factor the same as of the attachment $F$ in Fig. 2(a).

At last, we examine the infrared divergences from Fig. 3 (b) with the radiative gluon being emitted by the valence anti-quark of the $M_{2}$ meson and attaching to the other lines. The integrands for the attachments $A, D$ and $E$ contain the denominators

$$
\begin{aligned}
& {\left[\left(k_{1}+k_{2}+l\right)^{2}+i \epsilon\right]\left[\left(k_{2}+l\right)^{2}+i \epsilon\right]\left(l^{2}+i \epsilon\right)\left[\left(P_{B}-k+l\right)^{2}-m_{b}^{2}+i \epsilon\right]\left[\left(k-k_{1}-k_{2}-l\right)^{2}+i \epsilon\right],} \\
& {\left[\left(k_{1}+k_{2}+l\right)^{2}+i \epsilon\right]\left[\left(k_{2}+l\right)^{2}+i \epsilon\right]\left[(k+l)^{2}+i \epsilon\right]\left(l^{2}+i \epsilon\right),} \\
& {\left[\left(k_{1}+k_{2}+l\right)^{2}+i \epsilon\right]\left[\left(k_{2}+l\right)^{2}+i \epsilon\right]\left(l^{2}+i \epsilon\right)\left[\left(\bar{k}_{1}-l\right)^{2}+i \epsilon\right]\left[\left(k-k_{1}-k_{2}-l\right)^{2}+i \epsilon\right],}
\end{aligned}
$$

respectively. It is easy to show that these attachments do not produce Glauber divergences, simply because only the range $l^{-}<0$ contributes to the loop integrals. The attachments $B$ and $C$, located on the virtual lines, do not either. The attachment $F$, representing a vertex correction, is free of a Glauber divergence. That is, all the attachments in Fig. 3(b) contribute only to the $M_{2}$ meson wave function.

The NLO residual infrared divergence in the attachment $F$ in Fig. 3(a) is then extracted from the Glauber region and collected by the integral similar to Eq. (19). The argument leading to Eq. (21) also applies: since the poles $l^{+} \sim O\left(k_{1}^{+}\right)$in Eqs. (28) and (29) in the upper half plane stay far away from the selected pole $l^{+} \sim O\left(\Lambda^{2} / m_{B}\right)$, we can always deform the contour of $l^{+}$, such that the quark carrying the momentum $\bar{k}_{2}+l$ is eikonalized into $1 /\left(l^{+}+i \epsilon\right)$. We then obtain explicitly the imaginary logarithm:

$$
\frac{i}{2 N_{c}} \frac{\alpha_{s}}{2 \pi} \int \frac{d^{2} l_{T}}{l_{T}^{2}} \mathcal{M}_{b}^{(0)}\left(\mathbf{l}_{T}\right)
$$

where $\mathcal{M}_{b}^{(0)}$ represents the LO nonfactorizable annihilation amplitude from Fig. 1(b). It is noticed that Eq. (33) has a sign the same as of Eq. (22). This observation differs from that for the spectator amplitudes made in [15]: the Glauber logarithms for the two LO spectator amplitudes associated with the $M_{2}$ meson emitted at the weak vertex are opposite in sign.

The Glauber divergences associated with the $M_{1}$ meson are analyzed in the same way, and the results are similar to Eqs. (22) and (33). In this case a Glauber gluon is emitted by the valence anti-quark in the $M_{1}$ 
meson and attaches to the valence anti-quark in the $M_{2}$ meson, so the Glauber divergences have the same sign as Eqs. (22) and (33). The exponentiation of the NLO results in Eqs. (22) and (33) yields

$$
\begin{aligned}
& M_{a}^{G}=\exp \left(i S_{G}\right) \mathcal{M}_{a}^{(0)}, \\
& M_{b}^{G}=\exp \left(i S_{G}\right) \mathcal{M}_{b}^{(0)},
\end{aligned}
$$

where both the LO amplitudes have been rotated by the same Glauber phase $S_{G}$. Equation (34) concludes our investigation of the Glauber divergences in the nonfactorizable annihilation diagrams for the two-body hadronic heavy meson decays. It also holds for nonfactorizable $W$-exchange amplitudes, since fermion flows do not affect the derivation of the Glauber factors. A definition for the Glauber factor in terms of a matrix element of four Wilson links has been constructed in [13]. Strictly speaking, Eq. (34) should be understood as convolutions between the Glauber factors and the LO nonfactorizable annihilation amplitudes in the impact parameter space 17]. Nevertheless, treating $S_{G}$ as a constant parameter is convenient for phenomenological applications, such as the FAT parametrization for two-body hadronic heavy meson decays.

\section{PHENOMENOLOGICAL EFFECTS}

It has been shown that the nonfactorizable annihilation contribution is less than $10 \%$ of the factorizable one in two-body hadronic $B$ meson decays [29]. The Glauber factor obtained here introduces an overall phase to the nonfactorizale annihilation amplitude, instead of changing the interference pattern between the two LO hard diagrams. This contribution is thus expected to remain negligible, and does not have a significant impact on, say, the $B \rightarrow \pi \pi$ and $B \rightarrow \pi K$ decays. That is, the $B \rightarrow \pi \pi$ and $B \rightarrow \pi K$ puzzles mentioned before are resolved mainly by the Glauber effects on the spectator contributions [7, 15, 17, 18]. The Glauber factor in Eq. (34) may be crucial for a decay dominated by the tree annihilation topology, to which the factorizable annihilation contribution is suppressed by the helicity conservation. The nonfactorizale and factorizable annihilation contributions then become comparable, such that the rotation of the former can give an effect. The $B_{c} \rightarrow \pi K$, $\pi \eta$ and $\pi \eta^{\prime}$ decays, belonging to this category, are appropriate for probing the Glauber effect discussed in this work. However, these modes with small branching ratios about $10^{-7}-10^{-8}[30$ ] are not easily accessed.

$D$ meson decays provide another potential arena for testing the Glauber effect on the nonfactorizable annihilation contributions. Penguin amplitudes in these decays are usually tiny, and annihilation contributions are not really power suppressed compared to emission ones, because the $D$ meson mass is not much higher than the QCD scale. For the same reason, the nonfactorizable annihilation contribution may be comparable to the factorizable one. The Glauber factor $\exp \left(i S_{G}\right)$ has been introduced as a free parameter in the FAT approach to two-body hadronic $D$ meson decays, and associated with a final-state pion in the nonfactorizable annihilation and $W$-exchange channels. The global fits to the measured $D \rightarrow P P$ and $P V$ branching ratios confirmed that the Glauber phases are substantial, $S_{G}=-0.50$ [21] and $S_{G}=-0.85$ with the $\rho-\omega$ mixing being taken into account [31], respectively. The puzzle from the data of the $D^{0} \rightarrow \pi^{+} \pi^{-}$and $D^{0} \rightarrow K^{+} K^{-}$branching ratios stated in the Introduction was then resolved [21]. These sizable Glauber phases from the global analyses indicate that they are demanded by modes involving pions in general.

Below we manifest the Glauber effect in two-body hadronic $D$ meson decays from a different viewpoint: we identify several specific modes, whose data exhibit the impact of the Glauber phase clearly. Consider the doublyCabibbo-suppressed $D^{+} \rightarrow \pi^{0} K^{+}$and $D^{0} \rightarrow \pi^{-} K^{+}$decays, both of which involve the color-allowed emission amplitude with the $D \rightarrow \pi$ transition. The former (latter) also proceeds via the annihilation ( $W$-exchange) process with the $u \bar{u}$ quark pair popping out of the vacuum. It is expected that the properties of individual mesons which may break the SU(3) symmetry, such as the decay constants, cancel largely in the ratio of their branching fractions. The corresponding singly-Cabibbo-suppressed $D^{+} \rightarrow K_{S} K^{+}$and $D^{0} \rightarrow K^{-} K^{+}$decays involve the color-allowed emission amplitude with the $D \rightarrow K$ transition. The former (latter) also proceeds via the annihilation ( $W$-exchange) process with the $s \bar{s}(u \bar{u})$ quark pair popping out of the vacuum. The $\mathrm{SU}(3)$ symmetry breaking effects also cancel largely in the ratio of their branching fractions. Note that the emission and annihilation amplitudes in the $D^{+} \rightarrow \pi^{0} K^{+}$decay are opposite in sign, attributed to the $d \bar{d}(u \bar{u})$ component of the $\pi^{0}$ meson contributing to the former (latter). This opposite sign in the corresponding $D^{+} \rightarrow K_{S} K^{+}$decay is provided by the different Cabibbo-Kobayashi-Maskawa (CKM) factors associated with the two amplitudes. 
The similarity between the above $D \rightarrow \pi K$ and $D \rightarrow K K$ sets suggests

$$
\frac{B\left(D^{+} \rightarrow \pi^{0} K^{+}\right)}{B\left(D^{0} \rightarrow \pi^{-} K^{+}\right)} \approx \frac{B\left(D^{+} \rightarrow K_{S} K^{+}\right)}{B\left(D^{0} \rightarrow K^{-} K^{+}\right)}
$$

However, the data of the branching ratios $B\left(D^{+} \rightarrow \pi^{0} K^{+}\right)=(2.08 \pm 0.21) \times 10^{-4}, B\left(D^{0} \rightarrow \pi^{-} K^{+}\right)=$ $(1.50 \pm 0.07) \times 10^{-4}, B\left(D^{+} \rightarrow K_{S} K^{+}\right)=(3.04 \pm 0.09) \times 10^{-3}$ and $B\left(D^{0} \rightarrow K^{-} K^{+}\right)=(4.08 \pm 0.06) \times 10^{-3}[32]$ lead to $1.39 \pm 0.15$ for the left-hand side and $0.745 \pm 0.025$ for the right-hand side, which differ significantly. The error for the ratio is estimated by summing the fractional errors of the numerator and the denominator in quadrature. It is very difficult to accommodate the above data without the Glauber effect associated with a pion.

Another example comes from the comparison of the $D \rightarrow \pi \pi$ and $\pi K$ decays. Both the singly-Cabibbosuppressed $D^{0} \rightarrow \pi^{-} \pi^{+}$and $D^{0} \rightarrow \pi^{0} \pi^{0}$ decays proceed via the $W$-exchange process with the light (non-strange) quark pairs popping out of the vacuum. The former (latter) also involves the color-allowed (color-suppressed) emission amplitude with the $D \rightarrow \pi$ transition. The corresponding modes are the doubly-Cabibbo-suppressed $D^{0} \rightarrow \pi^{-} K^{+}$decay and the Cabibbo-favored $D^{0} \rightarrow \pi^{0} K_{S}$ decay, which involve the color-allowed and colorsuppressed emission amplitudes, respectively, and the $W$-exchange process with the light quark pairs popping out of the vacuum. The Cabibbo-favored $D^{0} \rightarrow \pi^{+} K^{-}$decay can be also considered, which results from the color-allowed emission amplitude with the $D \rightarrow K$ transition, and the $W$-exchange channel with the light quark pair popping out of the vacuum. The similarity among the above sets of modes implies

$$
\frac{B\left(D^{0} \rightarrow \pi^{-} \pi^{+}\right)}{B\left(D^{0} \rightarrow \pi^{0} \pi^{0}\right)} \approx \frac{B\left(D^{0} \rightarrow \pi^{-} K^{+}\right)}{\lambda^{4} B\left(D^{0} \rightarrow \pi^{0} K_{S}\right)} \approx \frac{B\left(D^{0} \rightarrow \pi^{+} K^{-}\right)}{B\left(D^{0} \rightarrow \pi^{0} K_{S}\right)}
$$

where the denominator of the second ratio has been corrected by the Wolfenstein parameter $\lambda=0.23$. Since the $W$-exchange amplitudes in all the four modes arise from the light quark pair production in the vacuum, the first equality is expected to hold better than Eq. (35). The two modes in the third ratio contain different transition form factors, but the $\mathrm{SU}(3)$ symmetry breaking effects in the total decay amplitudes still cancel to some extent. Hence, the second equality provides useful information, and is worth consideration.

The data $B\left(D^{0} \rightarrow \pi^{-} \pi^{+}\right)=(1.455 \pm 0.024) \times 10^{-3}, B\left(D^{0} \rightarrow \pi^{0} \pi^{0}\right)=(8.26 \pm 0.25) \times 10^{-4}, B\left(D^{0} \rightarrow\right.$ $\left.\pi^{-} K^{+}\right)=(1.50 \pm 0.07) \times 10^{-4}$ and $B\left(D^{0} \rightarrow \pi^{0} K_{S}\right)=(1.240 \pm 0.022) \times 10^{-2}$ lead to $1.76 \pm 0.06$ for the first ratio and $4.32 \pm 0.22$ for the second ratio in Eq. (36), which differ dramatically. The data $B\left(D^{0} \rightarrow \pi^{+} K^{-}\right)=$ $(3.950 \pm 0.031) \times 10^{-2}$ yield the third ratio $3.19 \pm 0.062$, which is closer to the second ratio. Note that the modes in the first ratio have more final-state pions than those in the second and third ratios do. The apparent difference between the first ratio and the second and third ratios further supports that the Glauber effect associated with final-state pions is necessary for explaining the $D \rightarrow P P$ data by differentiating the interference patterns between the emission and $W$-exchange amplitudes.

\section{CONCLUSION}

In this paper we have identified the residual Glauber divergences in the $k_{T}$ factorization for the nonfactorizable annihilation amplitudes in two-body hadronic heavy meson decays at NLO level. Radiative corrections to these amplitudes produce not only ordinary collinear logarithms, which are absorbed into final-state meson wave functions, but imaginary infrared logarithms, which demand the introduction of additional nonperturbative inputs. It has been shown that the Glauber divergences are factorizable in the region with small parton momenta, to which the $k_{T}$ factorizaton theorem applies. It was observed that the resultant phase factors for the two LO nonfactorizable annihilation diagrams are the same. Therefore, the Glauber gluon effect rotates these amplitudes, and modifies their interferences with other amplitudes, such that branching ratios and direct $\mathrm{CP}$ asymmetries of some two-body hadronic heavy meson decays can be changed. Besides, this work provides a solid theoretical ground for the FAT parametrization of two-body hadronic $D$ meson decays proposed a decade ago [21].

Because the Glauber phase factors are of perturbative origin, there is no a priori knowledge about their importance in heavy flavor decays. We have demonstrated that the data suggest prominent Glauber effects in pion-involving modes, which have been argued for by means of the simultaneous role of a pion as a $q \bar{q}$ bound state and a pseudo Nambu-Goldstone boson. The Glauber effects in two-body hadronic $B$ meson decays were crucial 
for resolving the $B \rightarrow \pi \pi$ and $\pi K$ puzzles. The similar observation has been made here for two-body hadronic $D$ meson decays into pions: the obvious difference among the measured $D \rightarrow \pi \pi, \pi K$ and $K K$ branching ratios reflects the Glauber effects associated with the final-state pions, which differentiate the interference patterns between the emission and annihilation ( $W$-exchange) amplitudes in the above modes. We have explained that

the Glauber divergences are absent in the collinear factorization for heavy meson decays. A derivation of the Glauber phases in the $k_{T}$ factorization by nonperturbative methods for various mesons will help verifying the mechanism elaborated in this work.

\section{Acknowledgments}

I thank X.D. Gao, X. Liu, S. Mishima, Y.H. Tsai, F.S. Yu and X. Yu for stimulating discussions. This work was supported in part by MOST of R.O.C. under Grant No. MOST-107-2119-M-001-035-MY3.

[1] J. Collins and J. W. Qiu, Phys. Rev. D 75, 114014 (2007); J. Collins, arXiv:0708.4410 [hep-ph].

[2] F. Liu and J. P. Ma, arXiv:0802.2973 [hep-ph]].

[3] C. W. Bauer, B. O. Lange and G. Ovanesyan, JHEP 07, 077 (2011).

[4] S. Fleming, Phys. Lett. B 735, 266-271 (2014).

[5] J. R. Gaunt, JHEP 07, 110 (2014).

[6] M. Diehl, J. R. Gaunt, D. Ostermeier, P. Plößl and A. Schäfer, JHEP 01, 076 (2016).

[7] H. n. Li and S. Mishima, Phys. Rev. D 83, 034023 (2011).

[8] C. Chiang et al., Phys. Rev. D 70, 034020 (2004); Y. Y. Charng and H. n. Li, Phys. Rev. D 71, 014036 (2005); R. Fleischer, S. Recksiegel and F. Schwab, Eur. Phys. J. C 51, 55 (2007).

[9] T. N. Pham, arXiv:0910.2561 [hep-ph].

[10] H. Y. Cheng and C. K. Chua, Phys. Rev. D 80, 074031 (2009).

[11] Y. Y. Keum, H. n. Li and A. I. Sanda, Phys Lett. B 504, 6 (2001); Phys. Rev. D 63, 054008 (2001).

[12] C. D. Lü, K. Ukai and M. Z. Yang, Phys. Rev. D 63, 074009 (2001).

[13] C. p. Chang and H. n. Li, Eur. Phys. J. C 71, 1687 (2011); H. n. Li, arXiv:1009.3610 [hep-ph].

[14] M. Nagashima and H. n. Li, Phys. Rev. D 67, 034001 (2003).

[15] H. n. Li and S. Mishima, Phys. Rev. D 90, 074018 (2014).

[16] G. P. Lepage and S. J. Brodsky, Phys. Lett. B 87, 359 (1979); S. Nussinov and R. Shrock, Phys. Rev. D 79, 016005 (2009); M. Duraisamy and A. L. Kagan, Eur. Phys. J. C 70, 921 (2010).

[17] X. Liu, H. n. Li and Z. J. Xiao, Phys. Rev. D 91, 114019 (2015).

[18] X. Liu, H. n. Li and Z. J. Xiao, Phys. Rev. D 93, 014024 (2016).

[19] B. Bhattacharya and J. L. Rosner, Phys. Rev. D 81, 014026 (2010).

[20] H. Y. Cheng and C. W. Chiang, Phys. Rev. D81, 074021 (2010).

[21] H. n. Li, C. D. Lü and F. S. Yu, Phys. Rev. D 86, 036012 (2012).

[22] R. Aaij et al. [LHCb], JHEP 07, 041 (2014).

[23] R. Aaij et al. [LHCb], Phys. Rev. Lett. 116, 191601 (2016).

[24] H. Y. Cheng and C. W. Chiang, Phys. Rev. D 85, 034036 (2012) [erratum: Phys. Rev. D 85, 079903 (2012)]; Phys. Rev. D 86, 014014 (2012).

[25] S. H. Zhou, Y. B. Wei, Q. Qin, Y. Li, F. S. Yu and C. D. Lu, Phys. Rev. D 92, no.9, 094016 (2015).

[26] H. n. Li, Y. L. Shen and Y. M. Wang, Phys. Rev. D 85, 074004 (2012).

[27] H. n. Li and B. Tseng, Phys. Rev. D 57, 443 (1998).

[28] M. Beneke, G. Buchalla, M. Neubert and C. T. Sachrajda, Phys. Rev. Lett. 83, 1914 (1999); Nucl. Phys. B 591, $313(2000)$.

[29] Y. Y. Keum and H. n. Li, Phys. Rev. D 63, 074006 (2001).

[30] X. Liu, Z. J. Xiao and C. D. Lu, Phys. Rev. D 81, 014022 (2010).

[31] H. n. Li, C. D. Lü, Q. Qin and F. S. Yu, Phys. Rev. D 89, 054006 (2014).

[32] P. A. Zyla et al. (Particle Data Group), Prog. Theor. Exp. Phys. 2020, 083C01 (2020). 